\title{
Image Simulations of Defect Images in STEM Observation Mode
}

\author{
M. De Graef
}

Department of Materials Science and Engineering, Carnegie Mellon University, Pittsburgh PA 15213

Defects in crystalline solids have traditionally been investigated by means of bright field/dark field imaging methods, often using the two-beam approximation. While the image formation mechanisms are well understood, and have been implemented in a number of defect image simulation programs, little attention has been devoted to defect observations under conical illumination conditions, i.e., STEM imaging conditions. This contribution reports on a simulation algorithm that employs the systematic row scattering matrix approach for the computation of bright field (BF) and annular dark field (ADF) detector signals for a thin foil, possibly curved, containing an arbitrary number of defects, including straight dislocations, stacking faults with bounding partials, spherical inclusions and voids. The algorithm takes into account the dimensions of the BF and ADF detectors, the microscope camera length, the beam convergence angle for STEM illumination and, if present, an objective aperture.

The algorithm is based on the observation that, in the Darwin-Howie-Whelan dynamical multi-beam equations, the phase shift corresponding to an arbitrary superposition of defects with combined displacement field $\mathbf{R}$ is a periodic function of the $\operatorname{argument} \alpha=2 \pi \mathbf{g} \cdot \mathbf{R}$, where $\mathbf{g}$ is the shortest reciprocal lattice vector of the systematic row [1]. Therefore, in the scattering matrix formalism one can pre-compute a large number of scattering matrices for a given diffraction condition (i.e., a given excitation error) and then select, via a simple modulo operation, the appropriate scattering matrix based on the local value of $\mathbf{g} \cdot \mathbf{R}$. Since matrix multiplication can be implemented efficiently, the resulting code is reasonably fast, even for large images (e.g., $1024 \times 1024)$. Note that, contrary to the standard differential equation solver approach used in such programs as the Head\&Humble dislocation simulation code [2], the present algorithm uses the displacement field itself, rather than its derivative.

For a systematic row, the diffraction condition does not change perpendicular to the row, so that only a single series of incident beam directions needs to be taken into account. The correct intensity contributions to the BF and ADF detectors can then be computed by means of weight factors that are proportional to the length of the chord normal to the systematic row inside the diffraction disk, as illustrated in Fig. 1(a-c). The weight factors for the $\mathrm{g}_{020}$ systematic row of copper (at $200 \mathrm{kV}$ ) with a beam divergence angle of $\theta_{c}=7 \mathrm{mrad}$, a microscope camera length of $L=400 \mathrm{~mm}$, are shown in Fig. 1(c). A total of 51 incident beam directions were used for the three example computations in Fig. 2. In (a) and (b), the bright field and $\mathbf{g}_{220}$ dark field images are shown for a slightly bent foil, 125 $\mathrm{nm}$ thick, with six random perfect dislocations, three stacking faults bounded by partials, fifty random voids and fifty random spherical inclusions. These images (field of view $1 \times 1 \mu \mathrm{m}^{2}$ ) were computed for a 7-beam systematic row with parallel illumination and $s_{220}=0$. Fig. 2(c) and (d) show the bright field and weak beam dark field $\left(s_{660}=0\right)$ images for the same defect configuration and parallel illumination. Fig. 2(e) and (f) show the BF and ADF images using illumination conditions similar to those shown in Fig. 1(c) $\left(L=350 \mathrm{~mm}, \theta_{c}=7 \mathrm{mrad}, s_{220}=0\right)$ for a 7-beam systematic row. Note how the diffraction contrast in between the defects has decreased significantly in the BF-STEM image, so that the defects are more clearly visible as compared to the conventional BF image in Fig. 2(a). Additional examples of STEM BF and ADF simulations for stacking faults and dislocations in Ni-based super alloys will be discussed. 


\section{References}

[1] M. De Graef, Intro. to Conventional Transmission Electron Microscopy, Cambridge Univ. Press (2003) . [2] A.K. Head, P. Humble, L.M. Clarebrough, A.J. Morton, and C.T. Forwood. Computed Electron Micrographs and Defect Identification, North Holland Publishing Company, 1973.

[3] The author would like to acknowledge stimulating discussions with P. Phillips and M. Mills (OSU). This work was supported by the National Science Foundation under grant \# DMR-0809048.
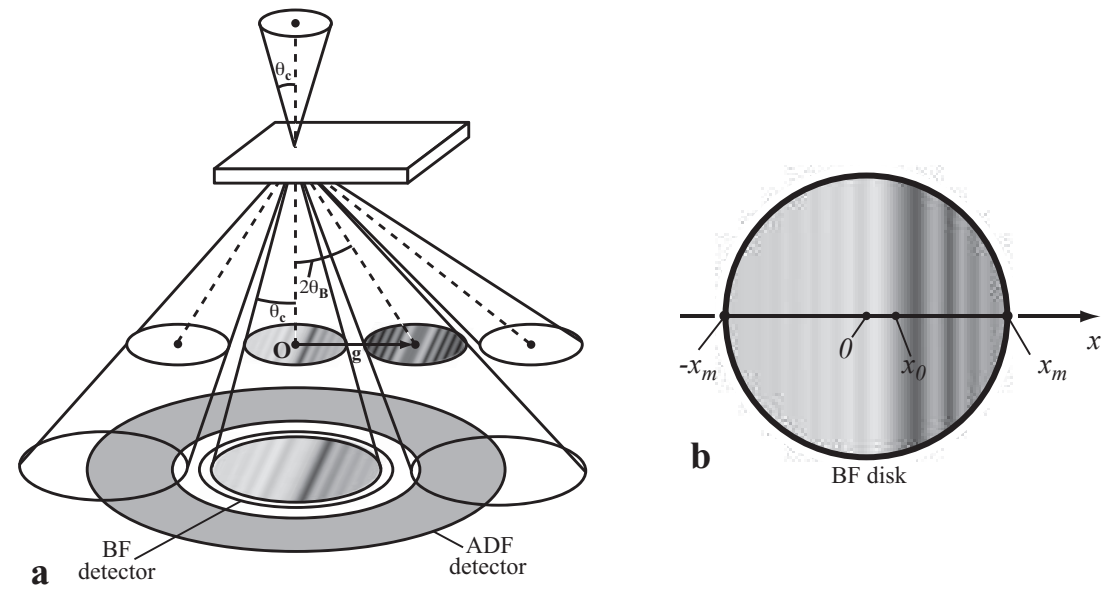

Figure 1: (a) schematic of systematic row in STEM illumination condition; (b) diffraction
stant normal to the systematic row; (c) BF and ADF weight factors for a systematic row.

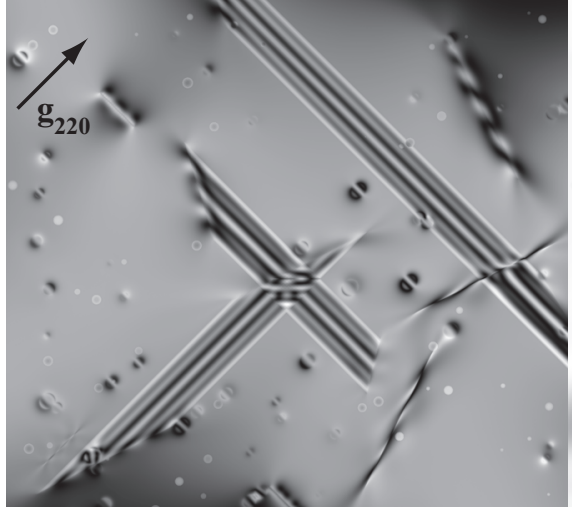

(a) $B F-C T E M$

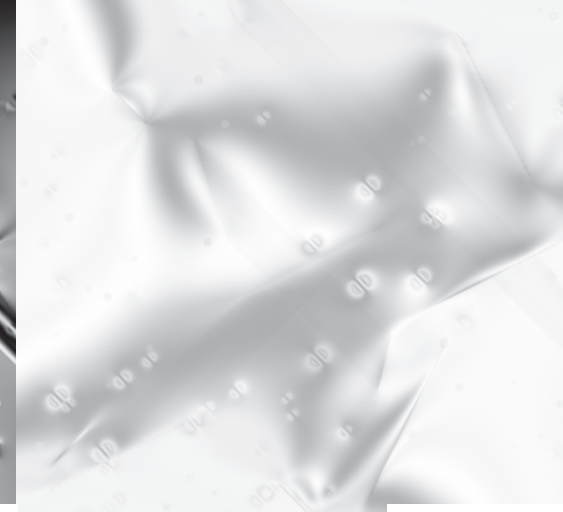

(c) $B F-W B$

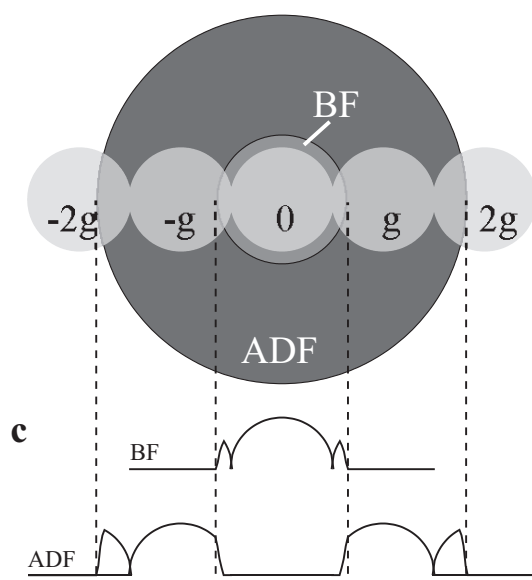

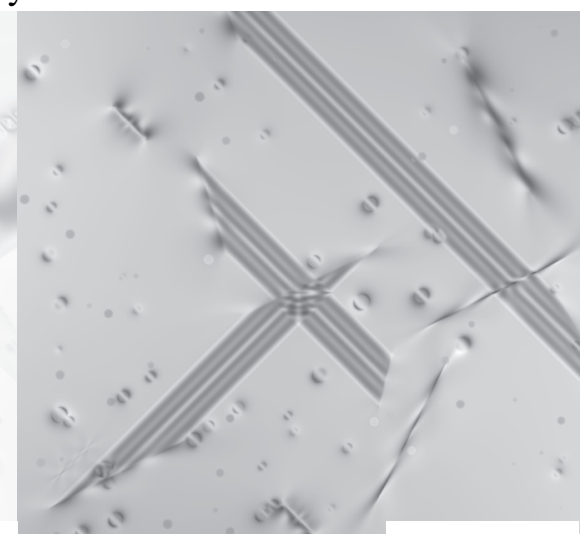

(e) $B F-S T E M$

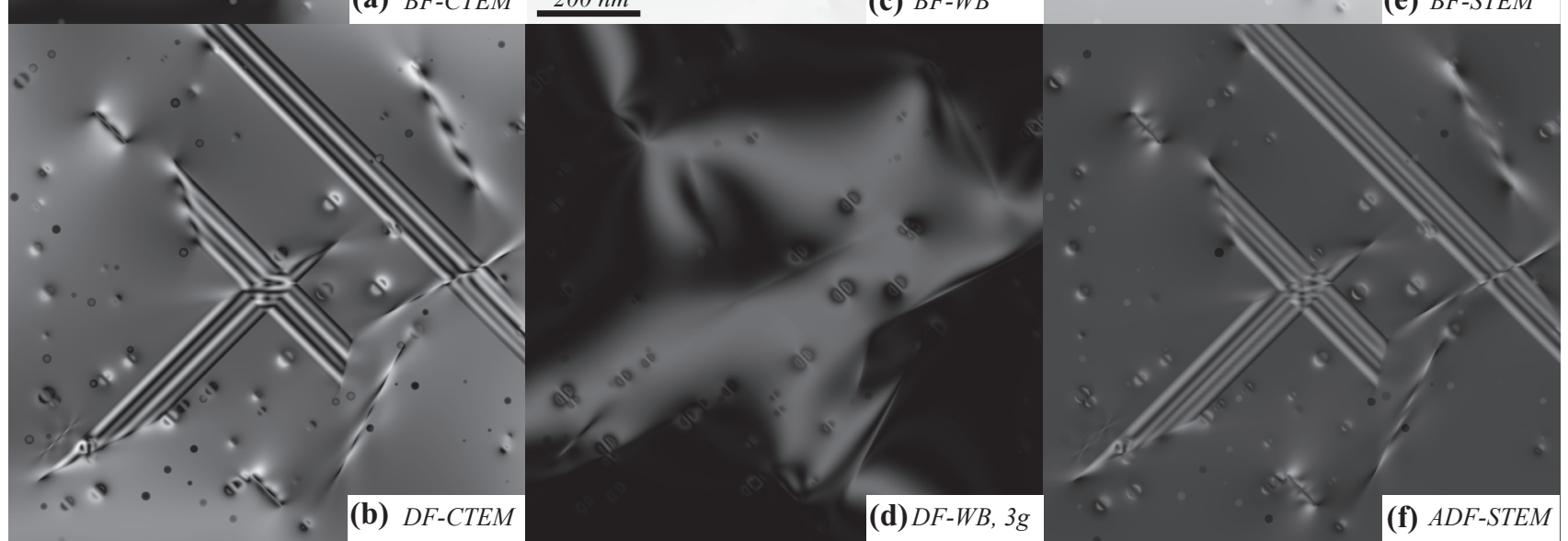

Figure 2: (a)-(b) simulated BF-DF pair with $s_{020}=0$; (c)-(d) simulated BF-DF pair in weak beam orientation $s_{060}=0$; (e)-(f) BF and ADF STEM images for $s_{020}=0$, using the detector geometry of Fig. 1(c). 